\title{
Bending and Vibrations of a Thick Plate with Consideration of Bimoments
}

\author{
Makhamatali K. Usarov, Davronbek M. Usarov, Gayratjon T. Ayubov \\ Institute of Seismic Stability of Structures of the Academy of Sciences of the Republic of Uzbekistan, Tashkent, \\ Uzbekistan \\ Email: umakhamatali@mail.ru
}

Received 1 July 2016; accepted 27 August 2016; published 30 August 2016

Copyright (C) 2016 by authors and Scientific Research Publishing Inc.

This work is licensed under the Creative Commons Attribution International License (CC BY). http://creativecommons.org/licenses/by/4.0/

c) (i) Open Access

\begin{abstract}
The paper is dedicated to the development of the theory of orthotropic thick plates with consideration of internal forces, moments and bimoments. The equations of motion of a plate are described by two systems of six equations. New equations of motion of the plate and the boundary conditions relative to displacements, forces, moments, and bimoments are given. As an example, the problems of free and forced oscillations of a thick plate are considered under the effect of sinusoidal periodic load. The problem is solved by Finite Difference Method. Eigenfrequencies of the plate are determined, numeric maximum values of displacements, forces and moments of the plate are obtained depending on the frequency of external force. It is shown that when the value of the frequency of external effect approaches the eigenfrequency, there occurs an increase in displacement, force and moment values; that testifies a gradual transition of the motion of plate points into the resonant mode.
\end{abstract}

\section{Keywords}

Plate, Orthotropic, Isotropic, Displacement, Stress, Moment, Bimoment, Bending, Vibrations

\section{Introduction}

Theory of plates and shells has a special place in design of structural elements. Specified theories of plates are built by many authors. All existing specified theories of plates are developed on the basis of a number of simplifying hypotheses. An overview of the main statements and common methods of constructing an improved theory of plates and shells can be found in the works of S. A. Ambartsumyan [1], K. Z. Galimov [2], Sh. K. Galimov [3], Kh. M. Mushtari [4] and others. Static problem of the bending of a thick isotropic plate in threedimensional theory of elasticity is considered by B.F. Vlasov in [5], which gives an exact analytical solution in 
trigonometric series. Monograph by E.N. Baida [6] is devoted to solving the problem of bending of orthotropic plates in trigonometric series. Numerical results of displacements and stresses are obtained.

The authors in [7]-[10] deal with dynamic problems of plates with anisotropic properties. Karamooz Ravari M.R., Forouzan M.R. [7] have studied the problems of plates oscillations. Frequency equations of orthotropic circular ring plate were obtained for general boundary conditions in oscillation plane. In [8] the solution of transition oscillations of rectangular viscous-elastic orthotropic plate are given for concrete strain models according to Flugge and Timoshenko-Mindlin's theories. The paper [9] is devoted to analytical solution of the problem of forced steady-state vibrations of orthotropic plate. By the method of superposition the problem is reduced to a quasi-regular infinite system of linear equations. In [10] an analytical method of solution of spatial problem of bending of orthotropic elastic plates subjected to external loads on upper and lower edges is developed. In [11] a problem is considered of a bending of orthotropic rectangular plate laying on two-parameter elastic foundation. Research in the field of thick plates has shown that in the case of spatial deformation of a plate along its thickness there occurs the nonlinear laws of displacements distribution and the hypothesis of plane sections is violated. In the cross-sections of the plate except for the tensile and shear forces, bending and torsional moments, there appear the additional force factors, the so-called bimoments. The author of the article addresses the problem of bending and vibrations of thick plates based on bimoment theory of plates [12]-[15], built as a part of three-dimensional theory of elasticity, using the method of displacements decomposition in one of the spatial coordinates in Maclaurin infinite series.

This paper gives a brief description of the technique of constructing a theory of plates with consideration of bimoments generated due to displacements distribution of cross-section points by a non-linear law. Here the equations of bimoments are built with the equation of three-dimensional dynamic theory of elasticity, described on face surfaces of the plate. The bimoments are introduced in stress dimensions and are characterized by the intensity of generated bimoments. We would use the designations and determinant correlations of forces, moments, bimoments and equations of motion relative to these force factors.

Unlike bimoment theory in [14] and [15], here the bimoment equations are built with the equation of threedimensional dynamic theory of elasticity, described on face surfaces of the plate. Bimoments are introduced in stress dimensions, and they characterize the intensity of generated bimoments.

Determinant relationships of forces, moments, bimoments and equations of motion relative to these force factors are given.

\section{Statement of the Problem}

Consider an orthotropic thick plate of constant thickness $H=2 h$ and dimensions $a, b$ in plane. Introduce the designations: $E_{1}, E_{2}, E_{3}$ - elasticity moduli; $G_{12}, G_{13}, G_{23}$-shear moduli; $v_{12}, v_{13}, v_{23}$-Poisson ratio of plate material.

When building an equation of motion the plate is considered as a three-dimensional body and all components of stress and strain tensors: $\sigma_{i j}, \varepsilon_{i j},(i, j=1,3)$ are taken into consideration. The components of displacement vector are the functions of three spatial coordinates and time $u_{1}\left(x_{1}, x_{2}, z, t\right), u_{2}\left(x_{1}, x_{2}, z, t\right), u_{3}\left(x_{1}, x_{2}, z, t\right)$.

The components of strain tensor $\varepsilon_{i j}$ are determined from Cauchy relation as:

$$
\begin{gathered}
\varepsilon_{11}=\frac{\partial u_{1}}{\partial x_{1}}, \varepsilon_{22}=\frac{\partial u_{2}}{\partial x_{2}}, \varepsilon_{33}=\frac{\partial u_{3}}{\partial z}, \\
\varepsilon_{12}=\frac{1}{2}\left(\frac{\partial u_{1}}{\partial x_{2}}+\frac{\partial u_{2}}{\partial x_{1}}\right), \varepsilon_{13}=\frac{1}{2}\left(\frac{\partial u_{1}}{\partial z}+\frac{\partial u_{3}}{\partial x_{1}}\right), \varepsilon_{23}=\frac{1}{2}\left(\frac{\partial u_{2}}{\partial z}+\frac{\partial u_{3}}{\partial x_{2}}\right),
\end{gathered}
$$

For orthotropic plate, the Hooke' law, in a general case, is written as:

$$
\begin{gathered}
\sigma_{11}=E_{11} \varepsilon_{11}+E_{12} \varepsilon_{22}+E_{13} \varepsilon_{33}, \\
\sigma_{22}=E_{21} \varepsilon_{11}+E_{22} \varepsilon_{22}+E_{23} \varepsilon_{33}, \\
\sigma_{33}=E_{31} \varepsilon_{11}+E_{32} \varepsilon_{22}+E_{33} \varepsilon_{33}, \\
\sigma_{12}=2 G_{12} \varepsilon_{12}, \quad \sigma_{13}=2 G_{13} \varepsilon_{13}, \quad \sigma_{23}=2 G_{23} \varepsilon_{23}
\end{gathered}
$$


where $E_{11}, E_{12}, \cdots, E_{33}$ are the elastic constants, determined through Poisson ratio and the moduli of elasticity in the form [14] [15].

As an equation of motion of a plate we would use three-dimensional equations of dynamic theory of elasticity:

$$
\begin{aligned}
& \frac{\partial \sigma_{11}}{\partial x_{1}}+\frac{\partial \sigma_{12}}{\partial x_{2}}+\frac{\partial \sigma_{13}}{\partial z}=\rho \ddot{\tilde{u}}_{2} \\
& \frac{\partial \sigma_{12}}{\partial x_{1}}+\frac{\partial \sigma_{22}}{\partial x_{2}}+\frac{\partial \sigma_{23}}{\partial z}=\rho \ddot{\tilde{u}}_{2} \\
& \frac{\partial \sigma_{13}}{\partial x_{1}}+\frac{\partial \sigma_{23}}{\partial x_{2}}+\frac{\partial \sigma_{33}}{\partial z}=\rho \ddot{\tilde{u}}_{3}
\end{aligned}
$$

where $\rho$ is a density of plate material.

Boundary conditions on lower and upper face surfaces of the plate $z=h$ and $z=-h$ are:

$$
\begin{aligned}
& \sigma_{33}=q_{3}^{(+)}, \quad \sigma_{31}=q_{1}^{(+)}, \sigma_{32}=q_{2}^{(+)}, \quad \text { at } z=h ; \\
& \sigma_{33}=q_{3}^{(-)}, \quad \sigma_{31}=q_{1}^{(-)}, \sigma_{32}=q_{2}^{(-)}, \quad \text { at } z=-h .
\end{aligned}
$$

Here $q_{1}^{(-)}, q_{2}^{(-)}, q_{3}^{(-)}$and $q_{1}^{(+)}, q_{2}^{(+)}, q_{3}^{(+)}$are distributed external loads, applied to upper and lower face surfaces of the plate $z=h$ and $z=-h$ along the direction of $o x_{1}, o x_{2}, o z$ coordinates axes.

\section{Method of Solution}

The methods of building the bimoment theory of plates are based on Cauchy relation (1), generalized Hooke's law (2), three-dimensional equations of the theory of elasticity (3), boundary conditions on face surfaces (4). A proposed bimoment theory of plates is also described by two non-connected problems, each of which is formulated on the basis of six two-dimensional equations of motion with corresponding boundary conditions.

The components of displacement vector are expanded into Maclaurin infinite series in the form:

$$
\begin{gathered}
u_{k}=B_{0}^{(k)}+B_{1}^{(k)} \frac{z}{h}+B_{2}^{(k)}\left(\frac{z}{h}\right)^{2}+B_{3}^{(k)}\left(\frac{z}{h}\right)^{3}+\cdots+B_{i}^{(k)}\left(\frac{z}{h}\right)^{i}, \quad(k=1,2) \\
u_{3}=A_{0}+A_{1} \frac{z}{h}+A_{2}\left(\frac{z}{h}\right)^{2}+A_{3}\left(\frac{z}{h}\right)^{3}+\cdots+A_{i}\left(\frac{z}{h}\right)^{i}
\end{gathered}
$$

Here $B_{i}^{(k)}, A_{i}$ are unknown functions of two spatial coordinates and time $B_{i}^{(k)}=B_{i}^{(k)}\left(x_{1}, x_{2}, t\right)$, $A_{i}=A_{i}\left(x_{1}, x_{2}, t\right)$. In a general case, these functions are determined according to the formulae:

$$
B_{i}^{(k)}=\frac{1}{i !} h^{i}\left(\frac{\partial^{i} u_{k}}{\partial z^{i}}\right)_{z=0}, \quad(k=1,2), \quad A_{i}=\frac{1}{i !} h^{i}\left(\frac{\partial^{i} u_{3}}{\partial z^{i}}\right)_{z=0}
$$

The displacements in stresses in upper $z=-h$ and lower points $z=h$ in plate fibers we would designate as $u_{i}^{(-)}, u_{i}^{(+)},(i=1,3)$ and $\sigma_{i j}^{(-)}, \sigma_{i j}^{(+)},(i=1,3 ; j=1,3)$.

The first problem of bimoment theory describes tension-compression and transverse reduction of the plate, and the second one - the bending and transverse shear of the plate. Determinant relationships and corresponding equations of motion of the plate in the first and second problems are briefly described below.

The first problem is described by the forces and bimoments with six generalized functions $\bar{\psi}_{1}, \bar{\psi}_{2}, \bar{u}_{1}, \bar{u}_{2}, \bar{r}, \bar{W}$, which are determined by relationships:

$$
\begin{gathered}
\bar{u}_{k}=\frac{u_{k}^{(+)}-u_{k}^{(-)}}{2}, \quad \bar{\psi}_{k}=\frac{1}{2 h} \int_{-h}^{h} u_{k} \mathrm{~d} z, \quad(k=1,2), \\
\bar{W}=\frac{u_{3}^{(+)}-u_{3}^{(-)}}{2}, \quad \bar{r}=\frac{1}{2 h^{2}} \int_{-h}^{h} u_{3} z \mathrm{~d} z
\end{gathered}
$$


Introduce the external loads for the first problem

$$
\bar{q}_{1}=\frac{q_{1}^{(+)}-q_{1}^{(-)}}{2}, \quad \bar{q}_{2}=\frac{q_{2}^{(+)}-q_{2}^{(-)}}{2}, \quad \bar{q}_{3}=\frac{q_{3}^{(+)}+q_{3}^{(-)}}{2}
$$

The expressions of longitudinal and tangential forces are written as [12]-[15]:

$$
\begin{gathered}
N_{11}=E_{11} H \frac{\partial \bar{\psi}_{1}}{\partial x_{1}}+E_{12} H \frac{\partial \bar{\psi}_{2}}{\partial x_{2}}+2 E_{13} \bar{W}, \quad N_{22}=E_{12} H \frac{\partial \bar{\psi}_{1}}{\partial x_{1}}+E_{22} H \frac{\partial \bar{\psi}_{2}}{\partial x_{2}}+2 E_{23} \bar{W} \\
N_{12}=N_{21}=G_{12}\left(H \frac{\partial \bar{\psi}_{1}}{\partial x_{2}}+H \frac{\partial \bar{\psi}_{2}}{\partial x_{1}}\right)
\end{gathered}
$$

The intensities of the bimoments $\bar{p}_{13}, \bar{p}_{23}$ from tangential stresses $\sigma_{13}, \sigma_{23}$ have the expressions

$$
\bar{p}_{13}=G_{13}\left(\frac{2\left(\bar{u}_{1}-\bar{\psi}_{1}\right)}{H}+\frac{\partial \bar{r}}{\partial x_{1}}\right), \quad \bar{p}_{23}=G_{23}\left(\frac{2\left(\bar{u}_{2}-\bar{\psi}_{2}\right)}{H}+\frac{\partial \bar{r}}{\partial x_{2}}\right)
$$

The intensity of the bimoment $\bar{p}_{33}$ from normal stress $\sigma_{33}$ is written in the form:

$$
\bar{p}_{33}=E_{31} \frac{\partial \bar{\psi}_{1}}{\partial x_{1}}+E_{32} \frac{\partial \bar{\psi}_{2}}{\partial x_{2}}+E_{33} \frac{2 \bar{W}}{H}
$$

The equations of motion relative to longitudinal and tangential forces and bimoments from tangential and normal stresses have the form [12]-[15]:

$$
\begin{gathered}
\frac{\partial N_{11}}{\partial x_{1}}+\frac{\partial N_{12}}{\partial x_{2}}+2 \bar{q}_{1}=\rho H \ddot{\bar{\psi}}_{1}, \quad \frac{\partial N_{21}}{\partial x_{1}}+\frac{\partial N_{22}}{\partial x_{2}}+2 \bar{q}_{2}=\rho H \ddot{\bar{\psi}}_{2} \\
\frac{\partial \bar{p}_{13}}{\partial x_{1}}+\frac{\partial \bar{p}_{23}}{\partial x_{2}}-\frac{2 \bar{p}_{33}}{H}+\frac{2 \bar{q}_{3}}{H}=\rho \ddot{\bar{r}}
\end{gathered}
$$

Note, that the expressions of force factors (9), (10), and hence, the equations of motion of the system (11), (12) is rigorously built. This system consists of three equations relative to six unknown functions $\bar{\psi}_{1}, \vec{\psi}_{2}, \bar{u}_{1}, \bar{u}_{2}, \bar{r}, \bar{W}$. As could be seen, three equations are missed. If in expressions (9.a) the terms $2 E_{13} \bar{W}, 2 E_{13} \bar{W}$ are omitted, then we would obtain two equations of motion of classic theory of plates in the form (11), since the equation of motion (12) becomes isolated and fail.

The second problem of bimoment theory consists of the equations for bending moments, torsional moments, shear forces relative to six kinematic functions $\tilde{\psi}_{1}, \tilde{\psi}_{2}, \tilde{u}_{1}, \tilde{u}_{2}, \tilde{r}, \tilde{W}$, determined by formulae:

$$
\begin{gathered}
\tilde{u}_{k}=\frac{u_{k}^{(+)}-u_{k}^{(-)}}{2}, \quad \tilde{\psi}_{k}=\frac{1}{2 h^{2}} \int_{-h}^{h} u_{k} z \mathrm{~d} z, \quad(k=1,2), \\
\tilde{W}=\frac{u_{3}^{(+)}+u_{3}^{(-)}}{2}, \quad \tilde{r}=\frac{1}{2 h} \int_{-h}^{h} u_{3} \mathrm{~d} z
\end{gathered}
$$

Introduce the generalized external loads for the second problem

$$
\tilde{q}_{1}=\frac{q_{1}^{(+)}+q_{1}^{(-)}}{2}, \quad \tilde{q}_{2}=\frac{q_{2}^{(+)}+q_{2}^{(-)}}{2}, \quad \tilde{q}_{3}=\frac{q_{3}^{(+)}-q_{3}^{(-)}}{2}
$$

Bending, torsional moments and shear forces, which are rigorously built, have the form [12]-[15]:

$$
M_{11}=\frac{H^{2}}{2}\left(E_{11} \frac{\partial \tilde{\psi}_{1}}{\partial x_{1}}+E_{12} \frac{\partial \tilde{\psi}_{2}}{\partial x_{2}}-E_{13} \frac{2(\tilde{r}-\tilde{W})}{H}\right)
$$




$$
\begin{gathered}
M_{22}=\frac{H^{2}}{2}\left(E_{12} \frac{\partial \tilde{\psi}_{1}}{\partial x_{1}}+E_{22} \frac{\partial \tilde{\psi}_{2}}{\partial x_{2}}-E_{23} \frac{2(\tilde{r}-\tilde{W})}{H}\right) \\
M_{12}=\frac{H^{2}}{2} G_{12}\left(\frac{\partial \tilde{\psi}_{1}}{\partial x_{2}}+\frac{\partial \tilde{\psi}_{2}}{\partial x_{1}}\right) \\
Q_{13}=G_{13}\left(2 \tilde{u}_{1}+H \frac{\partial \tilde{r}}{\partial x_{1}}\right), \quad Q_{23}=G_{23}\left(\tilde{u}_{2}+H \frac{\partial \tilde{r}}{\partial x_{2}}\right)
\end{gathered}
$$

The system of equations of motion of the second problem consists of two equations relative to bending, torsional moments and one equation relative to shear force and it is written in the form [12]-[15]:

$$
\begin{gathered}
\frac{\partial M_{11}}{\partial x_{1}}+\frac{\partial M_{12}}{\partial x_{2}}-Q_{13}=\frac{H^{2}}{2} \rho \ddot{\ddot{\psi}}_{1}-H \tilde{q}_{1}, \quad \frac{\partial M_{21}}{\partial x_{1}}+\frac{\partial M_{22}}{\partial x_{2}}-Q_{23}=\frac{H^{2}}{2} \rho \ddot{\ddot{\psi}}_{2}-H \tilde{q}_{2} \\
\frac{\partial Q_{13}}{\partial x_{1}}+\frac{\partial Q_{23}}{\partial x_{2}}=H \rho \ddot{\tilde{r}}-2 \tilde{q}_{3}
\end{gathered}
$$

Note, that the expressions of forces and moments (16), hence, the equations of motion of the system (17), (18) are rigorously built. Similar to the first problem, here three equations are missed. The system of equations of motion (17), (18) consists of three equations relative to six unknown functions $\tilde{\psi}_{1}, \tilde{\psi}_{2}, \tilde{u}_{1}, \tilde{u}_{2}, \tilde{r}, \tilde{W}$. If in expressions of forces and moments, into the equations of motion (17) and (18) conventionally introduce $\tilde{u}_{1}=3 \tilde{\psi}_{1}, \tilde{u}_{2}=3 \tilde{\psi}_{2}$ and $E_{13}=E_{23}=0$, and the shear modulus $G_{13}, G_{23}$ substitute for $k_{z} G_{13}, k_{z} G_{23}$, (where $k_{z}$ is a shear coefficient), then an equation of motion of plates could be obtained according to Timoshenko's theory.

To complete the systems (11), (12) and (17) and (18) it is necessary to build two more systems, with three equations in each. Write down three equations of motion of the theory of elasticity (3) on face surfaces of the plate $z=-h$ and $z=+h$. Adding and subtracting the equations of the theory of elasticity (3) on face surfaces of the plate $z=-h$ and $z=h$, and taking into account the Hooke's law (2), surface conditions (4) and designations (6), (7) and (13), (14), two independent systems with three equations in each could be obtained. The first of these systems describes the first problem and has the form:

$$
\begin{gathered}
\frac{\partial \bar{\sigma}_{11}}{\partial x_{1}}+\frac{\partial \bar{\sigma}_{12}}{\partial x_{2}}+\frac{\bar{\sigma}_{13}^{*}}{H}=\rho \ddot{\bar{u}_{1}}, \quad \frac{\partial \bar{\sigma}_{21}}{\partial x_{1}}+\frac{\partial \bar{\sigma}_{22}}{\partial x_{2}}+\frac{\bar{\sigma}_{23}^{*}}{H}=\rho \ddot{\bar{u}_{2}} \\
\frac{\partial \bar{q}_{1}}{\partial x_{1}}+\frac{\partial \bar{q}_{2}}{\partial x_{2}}+\frac{\bar{\sigma}_{33}^{*}}{H}=\rho \ddot{\bar{W}}
\end{gathered}
$$

Here the intensities of the bimoments $\bar{\sigma}_{11}, \bar{\sigma}_{22}, \bar{\sigma}_{12}$-under transverse reduction and tension-compression of the plate, generated due to $\sigma_{11}, \sigma_{22}, \sigma_{12}$ are:

$$
\bar{\sigma}_{i j}=\frac{\sigma_{i j}^{(+)}+\sigma_{i j}^{(-)}}{2}, \quad(i=1,2 ; j=1,2)
$$

$\bar{\sigma}_{13}^{*}, \bar{\sigma}_{23}^{*}, \bar{\sigma}_{33}^{*}$ are the intensities of the bimoments generated due to transverse stresses $\sigma_{13}, \sigma_{23}, \sigma_{33}$ :

$$
\frac{\bar{\sigma}_{3 k}^{*}}{H}=\frac{1}{2}\left(\frac{\partial \sigma_{k 3}^{(+)}}{\partial z}+\frac{\partial \sigma_{k 3}^{(-)}}{\partial z}\right), \quad(k=1,2), \quad \frac{\bar{\sigma}_{33}^{*}}{H}=\frac{1}{2}\left(\frac{\sigma_{33}^{(+)}}{\partial z}-\frac{\partial \sigma_{33}^{(-)}}{\partial z}\right)
$$

The second system of equations obtained from the equations of the theory of elasticity (3) is written in the form:

$$
\frac{\partial \tilde{\sigma}_{11}}{\partial x_{1}}+\frac{\partial \tilde{\sigma}_{12}}{\partial x_{2}}+\frac{\tilde{\sigma}_{13}^{*}}{H}=\rho \ddot{\tilde{u}_{1}}, \quad \frac{\partial \tilde{\sigma}_{21}}{\partial x_{1}}+\frac{\partial \tilde{\sigma}_{22}}{\partial x_{2}}+\frac{\tilde{\sigma}_{23}^{*}}{H}=\rho \ddot{\tilde{u}}_{2}
$$




$$
\frac{\partial \tilde{q}_{1}}{\partial x_{1}}+\frac{\partial \tilde{q}_{2}}{\partial x_{2}}+\frac{\tilde{\sigma}_{33}^{*}}{H}=\rho \ddot{\tilde{W}}
$$

Here $\tilde{\sigma}_{11}, \tilde{\sigma}_{22}, \tilde{\sigma}_{12}$ are the intensities of the bimoments under transverse bending and shear for the second problem generated due to the stresses $\sigma_{11}, \sigma_{22}, \sigma_{12}$ :

$$
\tilde{\sigma}_{i j}=\frac{\sigma_{i j}^{(+)}-\sigma_{i j}^{(-)}}{2}, \quad(i=1,2 ; j=1,2)
$$

The intensities of the bimoments $\tilde{\sigma}_{13}^{*}, \tilde{\sigma}_{23}^{*}, \tilde{\sigma}_{33}^{*}$, generated due to the stresses $\sigma_{33}, \sigma_{13}, \sigma_{23}$, under transverse shear and bending are written in the form:

$$
\frac{\tilde{\sigma}_{3 k}^{*}}{H}=\frac{1}{2}\left(\frac{\partial \sigma_{k 3}^{(+)}}{\partial z}-\frac{\partial \sigma_{k 3}^{(-)}}{\partial z}\right),(k=1,2), \frac{\tilde{\sigma}_{33}^{*}}{H}=\frac{1}{2}\left(\frac{\sigma_{33}^{(+)}}{\partial z}+\frac{\partial \sigma_{33}^{(-)}}{\partial z}\right)
$$

The intensities of the bimoments $\bar{\sigma}_{11}, \bar{\sigma}_{22}, \bar{\sigma}_{12}, \tilde{\sigma}_{11}, \tilde{\sigma}_{12}, \tilde{\sigma}_{22}$ are determined from Hooke's law (2) with consideration of the conditions on face surfaces $z=-h$ and $z=h$ (4) as:

$$
\begin{aligned}
& \bar{\sigma}_{11}=E_{11}^{*} \frac{\partial \bar{u}_{1}}{\partial x_{1}}+E_{12}^{*} \frac{\partial \bar{u}_{2}}{\partial x_{2}}+\frac{E_{13}}{E_{33}} \bar{q}_{3}, \quad \bar{\sigma}_{22}=E_{12}^{*} \frac{\partial \bar{u}_{1}}{\partial x_{1}}+E_{22}^{*} \frac{\partial \bar{u}_{2}}{\partial x_{2}}+\frac{E_{23}}{E_{33}} \bar{q}_{3}, \quad \bar{\sigma}_{12}=G_{12}\left(\frac{\partial \bar{u}_{1}}{\partial x_{2}}+\frac{\partial \bar{u}_{2}}{\partial x_{1}}\right) \\
& \tilde{\sigma}_{11}=E_{11}^{*} \frac{\partial \tilde{u}_{1}}{\partial x_{1}}+E_{12}^{*} \frac{\partial \tilde{u}_{2}}{\partial x_{2}}+\frac{E_{13}}{E_{33}} \tilde{q}_{3}, \tilde{\sigma}_{22}=E_{12}^{*} \frac{\partial \tilde{u}_{1}}{\partial x_{1}}+E_{22}^{*} \frac{\partial \tilde{u}_{2}}{\partial x_{2}}+\frac{E_{23}}{E_{33}} \tilde{q}_{3}, \quad \tilde{\sigma}_{12}=G_{12}\left(\frac{\partial \tilde{u}_{1}}{\partial x_{2}}+\frac{\partial \tilde{u}_{2}}{\partial x_{1}}\right)
\end{aligned}
$$

Here $E_{11}^{*}=E_{11}-\frac{E_{13}}{E_{33}} E_{31}, E_{22}^{*}=E_{22}-\frac{E_{23}}{E_{33}} E_{32}, E_{12}^{*}=E_{21}-\frac{E_{23}}{E_{33}} E_{31}$.

The expressions of the intensities of the bimoments $\bar{\sigma}_{13}^{*}, \bar{\sigma}_{23}^{*}, \bar{\sigma}_{33}^{*}$ are determined by the solution of the system of linear algebraic equations relative to coefficients of Maclaurin series $B_{2 i}^{(1)}, B_{2 i}^{(2)}, A_{2 i+1},(i=0,1,2, \cdots)$, which are obtained by the substitution of the series (5) into the conditions on face surfaces at $z=-h$ and $z=h$ (4) and designations (6), (7).

$$
\begin{aligned}
\bar{\sigma}_{13}^{*}= & G_{13}\left(60 \frac{\bar{\psi}_{1}-\bar{u}_{1}}{H}-12 \frac{\partial \bar{W}}{\partial x_{1}}\right)-\frac{G_{13}}{E_{33}} H \frac{\partial}{\partial x_{1}}\left(E_{31} \frac{\partial \bar{u}_{1}}{\partial x_{1}}+E_{32} \frac{\partial \bar{u}_{2}}{\partial x_{2}}-\bar{q}_{3}\right)+12 \bar{q}_{1} \\
\bar{\sigma}_{23}^{*}= & G_{23}\left(60 \frac{\bar{\psi}_{2}-\bar{u}_{2}}{H}-12 \frac{\partial \bar{W}}{\partial x_{2}}\right)-\frac{G_{23}}{E_{33}} H \frac{\partial}{\partial x_{2}}\left(E_{31} \frac{\partial \bar{u}_{1}}{\partial x_{1}}+E_{32} \frac{\partial \bar{u}_{2}}{\partial x_{2}}-\bar{q}_{3}\right)+12 \bar{q}_{2} \\
\bar{\sigma}_{33}^{*}= & E_{33}\left(420 \frac{\bar{r}}{H}-180 \frac{\bar{W}}{H}\right)-H E_{31} \frac{\partial}{\partial x_{1}}\left(\frac{\partial \bar{W}}{\partial x_{1}}-\frac{\bar{q}_{1}}{G_{13}}\right)-H E_{32} \frac{\partial}{\partial x_{2}}\left(\frac{\partial \bar{W}}{\partial x_{2}}-\frac{\bar{q}_{2}}{G_{23}}\right) \\
& -20\left(E_{31} \frac{\partial \bar{u}_{1}}{\partial x_{1}}+E_{32} \frac{\partial \bar{u}_{2}}{\partial x_{2}}-\bar{q}_{3}\right)
\end{aligned}
$$

The expressions of the intensities of the bimoments $\tilde{\sigma}_{13}^{*}, \tilde{\sigma}_{23}^{*}, \tilde{\sigma}_{33}^{*}$ are determined by the solution of the system of linear algebraic equations relative to coefficients of Maclaurin series $B_{2 i+1}^{(1)}, B_{2 i+1}^{(2)}, A_{2 i},(i=0,1,2, \cdots)$, which are obtained by the substitution of the series (5) into the conditions on the face surfaces at $z=-h$ and $z=h \quad$ (4) and designations (13), (14).

$$
\begin{gathered}
\tilde{\sigma}_{13}^{*}=G_{13}\left(420 \frac{\tilde{\psi}_{1}}{H}-180 \frac{\tilde{u}_{1}}{H}-20 \frac{\partial \tilde{W}}{\partial x_{1}}\right)-\frac{G_{13}}{E_{33}} H \frac{\partial}{\partial x_{1}}\left(E_{31} \frac{\partial \tilde{u}_{1}}{\partial x_{1}}+E_{32} \frac{\partial \tilde{u}_{2}}{\partial x_{2}}-\tilde{q}_{3}\right)+20 \tilde{q}_{1}, \\
\tilde{\sigma}_{23}^{*}=G_{23}\left(420 \frac{\tilde{\psi}_{2}}{H}-180 \frac{\tilde{u}_{2}}{H}-20 \frac{\partial \tilde{W}}{\partial x_{2}}\right)-\frac{G_{23}}{E_{33}} H \frac{\partial}{\partial x_{2}}\left(E_{31} \frac{\partial \tilde{u}_{1}}{\partial x_{1}}+E_{32} \frac{\partial \tilde{u}_{2}}{\partial x_{2}}-\tilde{q}_{3}\right)+20 \tilde{q}_{2},
\end{gathered}
$$




$$
\begin{aligned}
\tilde{\sigma}_{33}^{*}= & 60 E_{33} \frac{\tilde{r}-\tilde{W}}{H}-E_{31} H \frac{\partial}{\partial x_{1}}\left(\frac{\partial \tilde{W}}{\partial x_{1}}-\frac{\tilde{q}_{1}}{G_{31}}\right)-E_{32} H \frac{\partial}{\partial x_{2}}\left(\frac{\partial \tilde{W}}{\partial x_{2}}-\frac{\tilde{q}_{2}}{G_{32}}\right) \\
& -12\left(E_{31} \frac{\partial \tilde{u}_{1}}{\partial x_{1}}+E_{32} \frac{\partial \tilde{u}_{2}}{\partial x_{2}}-\tilde{q}_{3}\right) .
\end{aligned}
$$

Write down the formulae to determine the displacements on the face surfaces of the plate $z=-h$ and $z=+h$ :

$$
u_{i}^{(-)}=\bar{u}_{i}-\tilde{u}_{i}, \quad u_{i}^{(+)}=\bar{u}_{i}+\tilde{u}_{i}, \quad(i=1,2), \quad u_{3}^{(-)}=\tilde{W}-\bar{W}, \quad u_{3}^{(+)}=\tilde{W}+\bar{W} .
$$

Formulae for stresses on the face surfaces of the plate $z=-h$ and $z=h$ have the form:

$$
\sigma_{i j}^{(-)}=\bar{\sigma}_{i j}-\tilde{\sigma}_{i j}, \quad \sigma_{i j}^{(+)}=\bar{\sigma}_{i j}+\tilde{\sigma}_{i j}, \quad(i=1,2 ; j=1,2)
$$

Maximum values of displacements and stresses of the plate are reached on the face surfaces of the plate and are determined by the solutions of the first and second problems by the formulae (33) and (34).

Note, that the expressions of intensities of the bimoments (10), (27), (28), (29), (30), (31) and (32) are built for the first time and are new in the theory of plates.

Consider the boundary conditions of a discussed problem for the thick plates.

1) On the border of the plate the displacements are zero. On the edges of the plate $x_{1}=$ const and $x_{2}=$ const the conditions should be as follows:

$$
\begin{gathered}
\bar{\psi}_{1}=0, \quad \bar{\psi}_{2}, \quad \bar{r}=0, \quad \bar{u}_{1}=0 ; \quad \bar{u}_{2}=0, \quad \bar{W}=0 \\
\tilde{\psi}_{1}=0, \quad \tilde{\psi}_{2}=0, \quad \tilde{r}=0, \quad \tilde{u}_{1}=0 ; \quad \tilde{u}_{2}=0, \quad \tilde{W}=0
\end{gathered}
$$

2) On the border $x_{1}=$ const the plate is supported. The following conditions should be satisfied:

$$
\begin{aligned}
& N_{11}=0, \quad N_{12}=0, \quad \bar{r}=0, \quad \bar{\sigma}_{11}=0, \quad \bar{\sigma}_{12}=0, \quad \bar{W}=0 \\
& M_{11}=0, \quad M_{12}=0, \quad \tilde{r}=0, \quad \tilde{\sigma}_{11}=0, \quad \tilde{\sigma}_{12}=0 \quad \tilde{W}=0
\end{aligned}
$$

3) On the border $x_{1}=$ const the plate is free of supports. The following conditions should be satisfied

$$
\begin{aligned}
& N_{11}=0, \quad N_{12}=0, \quad \bar{p}_{13}=0, \quad \bar{\sigma}_{11}=0, \quad \bar{\sigma}_{12}=0, \quad \bar{\sigma}_{13}^{*}=0 \\
& M_{11}=0, \quad M_{12}=0, \quad Q_{13}=0, \quad \tilde{\sigma}_{11}=0, \quad \tilde{\sigma}_{12}=0 \quad \tilde{\sigma}_{13}^{*}=0
\end{aligned}
$$

Boundary conditions on the border $x_{2}=$ const are similarly written.

When studying the problem of transverse bending and shear it is enough to consider only the second problem with the equations of motion (17), (18), (23), (24) and boundary conditions (35)-(40).

\section{Solution of Tests Problem}

As an example, consider the forced harmonic vibrations of a cantilever rectangular plate fixed on both ends under the effect of harmonic periodic external load:

$$
q_{1}^{(-)}=0, q_{2}^{(-)}=0, q_{1}^{(+)}=0, q_{2}^{(+)}=0, q_{3}^{(+)}=0, q_{3}^{(-)}=-q_{0} \sin \frac{\pi x_{1}}{a} \sin \frac{\pi x_{2}}{b} \sin \left(\omega_{0} t+\beta_{0}\right)
$$

where $q_{0}, \omega_{0}, \beta_{0}$ is an amplitude, frequency and the mode of vibration of an external load, respectively. Note, that if $\omega_{0}=0$, we obtain the problem of static bending of the plate.

Substituting (41) into (8) and (15) determine the load terms of the equation of motion. For a plate fixed on both ends the boundary conditions are written in the form (35) and (36).

\section{Numeric Results}

First determine eigenfrequencies of the plate. After dividing the variables by spatial coordinates and time, the 

problem is solved by Finite Difference Method. The step in spatial coordinates is $\Delta x_{1}=\Delta x_{2}=\frac{a}{30}$. In calcula-
tions, for isotropic plates $v_{21}=v_{13}=v_{32}=0.3$ are given as an initial data.

For square plates with dimensions $a=b=3 H$ the value of eigenfrequency is $p_{1}=0.7469$. With increasing dimensions of the plate up to $a=b=5 H$ the value of eigenfrequency is $p_{1}=0.3906$. For square plates with dimensions $a=b=8 H$ the value of eigenfrequency is $p_{1}=0.1983$.

Table 1 shows the results obtained for the displacements, moments and forces in fixed square plates $a=b=3 H$ under different values of dimensionless frequency $\bar{\omega}_{0}=\frac{\rho H^{2} \omega_{0}}{E_{1}}$. When the value of the frequency of external effect $\omega_{0}$ approaches the eigenfrequency $p_{1}=0.7469$ the values of the displacements, forces and moments dramatically increase; this testifies of gradual transition of the motion of plate points into resonant mode. As seen, an abrupt increase in the values of displacements, forces and moments could be observed.

Table 2 and Table 3 show numeric values of displacements, moments and forces, calculated for the fixed square plates with dimensions $a=b=5 H$ and $a=b=8 H$, respectively, for different values of dimensionless frequency $\bar{\omega}_{0}$.

Calculations show that when the value of the frequency of external effect $\omega_{0}$ approaches eigenfrequency, an increase in the values of displacements, forces and moments is observed; this testifies of gradual transition of the motion of plate points into resonant mode.

Table 1. Displacements, forces and moments at $a=b=3 H$.

\begin{tabular}{cccccc}
\hline $\bar{\omega}_{0}$ & $\frac{\tilde{\psi}_{1} E_{1}}{H q_{0}}$ & $\frac{\tilde{r} E_{1}}{H q_{0}}$ & $\frac{\tilde{W} E_{1}}{H q_{0}}$ & $M_{11}$ & $Q_{13}$ \\
\hline 0.0000 & -0.0158 & 0.8560 & 0.9402 & 0.0113 & 0.4591 \\
0.3000 & -0.0190 & 0.9882 & 1.0769 & -0.0108 & 0.5277 \\
0.4000 & -0.0226 & 1.1302 & 1.2240 & -0.0356 & 0.6010 \\
0.5000 & -0.0294 & 1.4039 & 1.5074 & -0.0852 & 0.7419 \\
0.6000 & -0.0463 & 2.0663 & 2.1937 & -0.2098 & 1.0816 \\
0.7000 & -0.1365 & 5.5665 & 5.8213 & -0.8909 & 2.8704 \\
\hline
\end{tabular}

Table 2. Displacements, forces and moments at $a=b=5 H$.

\begin{tabular}{cccccc}
\hline $\bar{\omega}_{0}$ & $\frac{\tilde{\psi}_{1} E_{1}}{H q_{0}}$ & $\frac{\tilde{r} E_{1}}{H q_{0}}$ & $\frac{\tilde{W} E_{1}}{H q_{0}}$ & $M_{11}$ & $Q_{13}$ \\
\hline 0.0000 & -0.0751 & 2.6428 & 2.7386 & -0.1047 & 0.7774 \\
0.1000 & -0.0802 & 2.7835 & 2.8825 & -0.1263 & 0.8155 \\
0.2000 & -0.1007 & 3.3478 & 3.4601 & -0.2158 & 0.9676 \\
0.3000 & -0.1783 & 5.4420 & 5.6053 & -0.5650 & 1.5275 \\
\hline
\end{tabular}

Table 3. Displacements, forces and moments at $a=b=8 H$.

\begin{tabular}{cccccc}
\hline $\bar{\omega}_{0}$ & $\frac{\tilde{\psi} E_{1}}{H q_{0}}$ & $\frac{\tilde{r} E_{1}}{H q_{0}}$ & $\frac{\tilde{W} E_{1}}{H q_{0}}$ & $M_{11}$ & $Q_{13}$ \\
\hline 0.0000 & -0.3067 & 8.3941 & 8.5060 & -0.3487 & 1.2598 \\
0.1000 & -0.3993 & 10.4739 & 10.6163 & -0.6033 & 1.5244 \\
0.1300 & -0.5105 & 12.9496 & 13.1296 & -0.9163 & 1.8364 \\
0.1600 & -0.8082 & 19.5290 & 19.8115 & -1.7691 & 2.6596 \\
0.1700 & -1.0499 & 24.8541 & 25.2206 & -2.4678 & 3.3234 \\
0.1800 & -1.5560 & 35.9866 & 36.5297 & -3.9369 & 4.7087 \\
\hline
\end{tabular}


Calculations show that the equations of motion of a plate (23) may be substituted by kinematic conditions relative to tangential stresses:

$$
\tilde{\sigma}_{13} \equiv G_{31}\left(12 \frac{\tilde{u}_{1}}{H}-30 \frac{\tilde{\psi}_{1}}{H}+\frac{\partial \tilde{W}}{\partial x_{1}}\right)=\tilde{q}_{1}, \quad \tilde{\sigma}_{23} \equiv G_{32}\left(12 \frac{\tilde{u}_{2}}{H}-30 \frac{\tilde{\psi}_{2}}{H}+\frac{\partial \tilde{W}}{\partial x_{2}}\right)=\tilde{q}_{2},
$$

Kinematic equations serve to determine the generalized displacements $\tilde{u}_{1}, \tilde{u}_{2}$.

The equations (26) are determined by the solution of the system of linear algebraic equations relative to coefficients of the series (5) $B_{2 i+1}^{(1)}, B_{2 i+1}^{(2)}, A_{2 i},(i=0,1,2, \cdots)$, which are obtained by the substitution of the series (5) into the conditions on the face surfaces at $z=-h$ and $z=h$ (4) and designations (13), (14).

\section{Conclusion}

Based on these studies, we would note that using the method of expansion in a series as part of three-dimensional dynamic theory of elasticity, a two-dimensional bimoment theory of orthotropic thick plates was developed and the equations of motion of the plate relative tot forces, moments and bimoments were built. It is shown that the problem in the general case is reduced to the definition of twelve unknown functions of two spatial coordinates and time. New expressions to determine the forces, moments and bimoments of the plates were built, as well as the methods for solving the problems of free and forced vibrations of plates based on Finite Difference Method.

\section{References}

[1] Ambartsumyan, S.A. (1987) Theory of Anisotropic Plates. Nauka, Ch. Ed. Sci. Lit., Moscow, 360 p.

[2] Galimov, K.Z. (1977) Theory of Shells with Account of Transverse Shear. Ed. Kazan University, Kazan, 212 p.

[3] Galimov, Sh.K. (1976) Specified Theory of Calculation of Orthotropic Rectangular Plate under Lateral Load. Investigations in Theory of Plates and Shells, Sat. articles, Kazan, Vol. XII, 78-84.

[4] Mushtari, Kh.M. (1990) Nonlinear Theory of Shells. Nauka, Moscow, 223 p.

[5] Vlasov, B.F. (1952) On a Case of Bending of a Rectangular Thick Plate. Vestnik MGU. Mechanics, Mathematics, Astronomy and Chemistry, No. 2, 25-34.

[6] Baida, E.N. (1983) Some Spatial Problems of Elasticity. Leningrad University, Leningrad, 232 p.

[7] Karamooz Ravari, M.R. and Forouzan, M.R. (2011) Frequency Equations for the In-Plane Vibration of Orthotropic Circular Annular Plate. Archive of Applied Mechanics, 81, 1307-1322.

[8] Soukup, J., Valeš, F., Volek, J. and Skočilas, J. (2011) Transient Vibration of thin Viscoelastic Orthotropic Plates. Acta Mechanica Sinica, 27, 98-107.

[9] Papkov, S.O. (2013) Steady-State Forced Vibrations of a Rectangular Orthotropic Plate. Journal of Mathematical Sciences, 192, 691-702.

[10] Chang, H.-H. and Tarn, J.-Q. (2012) Three-Dimensional Elasticity Solutions for Rectangular Orthotropic Plates. Journal of Elasticity, 108, 49-66.

[11] Zenkour, A.M., Allam, M.N.M., Shaker, M.O. and Radwan, A.F. (2011) On the Simple and Mixed First-Order Theories for Plates Resting on Elastic Foundations. Acta Mechanica, 220, 33-46.

[12] Usarov, M.K. (2014) Calculation of Orthotropic Plates Based on the Theory of Bimoments. Uzbek Journal Problems of Mechanics, Tashkent, No. 3-4, 37-41.

[13] Usarov, M.K. (2014) Bimoment Theory of Bending and Vibrations of Orthotropic Thick Plates. Vestnik NUU, No. 2/1, 127-132.

[14] Usarov, M.K. (2015) Bending of Orthotropic Plates with Consideration of Bimoments. St. Petersburg, Civil Engineering Journal, 1, 80-90.

[15] Usarov, M.K. (2015) On Solution of the Problem of Bending of Orthotropic Plates on the Basis of Bimoment Theory. Open Journal of Applied Sciences, 5, 212-219. http://dx.doi.org/10.4236/ojapps.2015.55021 


\section{Submit or recommend next manuscript to SCIRP and we will provide best service for you:}

Accepting pre-submission inquiries through Email, Facebook, LinkedIn, Twitter, etc.

A wide selection of journals (inclusive of 9 subjects, more than 200 journals)

Providing 24-hour high-quality service

User-friendly online submission system

Fair and swift peer-review system

Efficient typesetting and proofreading procedure

Display of the result of downloads and visits, as well as the number of cited articles

Maximum dissemination of your research work

Submit your manuscript at: http://papersubmission.scirp.org/ 